\section{Saumen Datta}

N. Shamala

Department of Physics

Indian Institute of Science

Bangalore-560012, India

Arindam Banerjee

P. Balaram

Molecular Biophysics Unit

Indian Institute of Science

Bangalore-560012, India

\section{Conformational Variability of Gly-Gly Segments in Peptides: A Comparison of the Crystal Structures of an Acyclic Pentapeptide and an Octapeptide}

The crystal structure of an acyclic pentapeptide, Boc-Gly-Gly-Leu-Aib-Val-OMe, reveals an extended conformation for the Gly-Gly segment, in contrast to the helical conformation determined earlier in the octapeptide Boc-Leu-Aib-Val-Gly-Gly-Leu-Aib-Val-OMe [I. L. Karle, A. Banerjee, S. Bhattacharjya, and P. Balaram [1996] Biopolymers, Vol. 38, pp. 515-526). The pentapeptide crystallizes in space group $P 2_{1}$ with one molecule in the asymmetric unit. The cell parameters are: $\mathrm{a}=10.979(2) \AA, \mathrm{b}=9.625(2) \AA, \mathrm{c}=14.141(2) \AA$, and $\beta=96.93(1)^{\circ}$, $\mathrm{R}=6.7 \%$ for 2501 reflections $(\mathrm{I}>3 \sigma(\mathrm{I}))$. The Gly-Gly segment is extended $\left(\phi_{1}=-92^{\circ}, \psi_{1}\right.$ $\left.=-133^{\circ}, \phi_{2}=140^{\circ}, \psi_{2}=170^{\circ}\right)$, while the Leu-Aib segment adopts a type II $\beta$-turn conformation $\left(\phi_{3}=-61^{\circ}, \psi_{3}=130^{\circ}, \phi_{4}=71^{\circ}, \psi_{4}=6^{\circ}\right)$. The observed conformation for the pentapeptide permits rationalization of a structural transition observed for the octapeptide in solution. An analysis of Gly-Gly segments in peptide crystal structures shows a preference for either $\beta$-turn or extended conformations. (C) 1997 John Wiley \& Sons, Inc.

\section{INTRODUCTION}

Crystal structure determinations of short acyclic peptides provide an opportunity to characterize specific conformational states in flexible molecules. ${ }^{1}$ Comparison of the solid state conformation of the same sequence segments determined under different conditions can provide information on the nature of conformational excursions that are possible. Investigation of synthetic peptides containing amino acid residues with contrasting conformational tendencies affords a means of evaluating the role of sequence and environmental effects. ${ }^{2,3}$ We have been investigating synthetic peptides containing Gly and Aib residues, the former being the least sterically de- manding of amino acid residues while the latter is the prototype of conformationally constrained residues having a very strong helix stabilizing tendency. ${ }^{4,5}$ In an earlier report we described the helical conformation in crystals of the octapeptide Boc-Leu-Aib-Val-Gly-Gly-Leu-Aib-Val-OMe, in which the central Gly-Gly segment is comfortably accommodated at the center of the helix. ${ }^{3}$ In solution, the Gly-Gly containing helix proved fragile, and unfolds in a strongly solvating medium like dimethylsulfoxide. In this paper, we describe the crystal structure of the protected pentapeptide fragment Boc-Gly-Gly-Leu-Aib-Val-OMe, in which a Leu-Aib type II $\beta$-turn is established, with the Gly-Gly arm adopting an extended conformation. A comparison of the pentapeptide and 
Table I Diffraction Data for Boc-Gly-Gly-Leu-Aib-Val-OMe

\begin{tabular}{ll}
\hline Empirical formula & $\mathrm{C}_{25} \mathrm{H}_{45} \mathrm{~N}_{5} \mathrm{O}_{8}$ \\
Crystal habit & $\mathrm{Clear}$ needle shaped \\
Crystal size $(\mathrm{mm})$ & $0.8 \times 0.2 \times 0.2$ \\
Crystallizing solvent & $\mathrm{CH}_{3} \mathrm{OH} / \mathrm{H}_{2} \mathrm{O}$ \\
Space group & $\mathrm{P} 2_{1}$ \\
Cell parameters & \\
$a(\AA)$ & $10.979(2)$ \\
$b(\AA)$ & $9.625(2)$ \\
$c(\AA)$ & $14.141(2)$ \\
$\beta\left({ }^{\circ}\right)$ & $96.93(1)$ \\
Volume $\left(\AA^{3}\right)$ & 1483.4 \\
$Z$ & 2 \\
Molecules/asymmetric unit & 1 \\
Molecular weight & 543.65 \\
Density $\left(\mathrm{g} / \mathrm{cm}{ }^{3} ;\right.$ calc. $)$ & 1.201 \\
$F(000)$ & 588 \\
Radiation $(\AA)$ & $\mathrm{CuK}(\lambda=1.5418)$ \\
Temperature $\left({ }^{\circ} \mathrm{C}\right)$ & 21 \\
$2 \theta$ Range $($ deg$)$ & 75 \\
Scan type & $\omega-2 \theta$ \\
Scan speed & Variable \\
Independent reflections & 2990 \\
Observed reflections. $[|F|>3 \sigma(F)]$ & 2501 \\
Final $R(\%)$ & 6.7 \\
Final $R_{\mathrm{w}}(\%)$ & 6.3 \\
Data-to-parameter ratio & $6.5: 1$ \\
$\Delta \rho_{\text {max }}\left(\mathrm{e} \AA^{-3}\right)$ & 0.31 \\
$\Delta \rho_{\text {min }}\left(\mathrm{e} \AA^{-3}\right)$ & -0.42 \\
Goodness of fit $(S)$ & 0.86 \\
\hline & \\
\hline & \\
&
\end{tabular}

octapeptide structures provides a means of rationalizing observed conformational changes in the latter.

\section{EXPERIMENTAL PROCEDURES}

Crystals of Boc-Gly-Gly-Leu-Aib-Val-OMe were grown from a methanol/water solution by slow evaporation. The crystals were transparent and needle shaped. The X-ray diffraction data were collected from a dry crystal on an automated four-circle diffractometer with $\mathrm{CuK}_{\alpha}$ radiation. Refined unit cell parameters were obtained by a leastsquares fit of the angular settings of 25 accurately determined reflections in the range $0^{\circ}<\theta<25^{\circ}$. Threedimensional intensity data were collected upto $2 \theta=75^{\circ}$ using $\omega-2 \theta$ scans, with variable scan speed. Two reflections used as standards, monitored after every 100 measurements, remained constant within 3\%. Pertinent parameters concerning data collection and the crystal are listed in Table I. The structure was obtained by direct methods using MULTAN-87. ${ }^{6}$ Refinement was carried out with a full-matrix least-squares method using
SHELX-76. ${ }^{7}$ Full matrix, anisotropic least-squares refinement was done on all the nonhydrogen atoms before fixing hydrogen. The hydrogen atoms were fixed geometrically in idealized positions, with $\mathrm{C}-\mathrm{H}=1.08 \AA$ and $\mathrm{N}-\mathrm{H}=1.08 \AA$, and refined as riding over the heavier atom to which they are bonded. The final $R$ factor was $6.7 \%\left(R_{w}=6.3 \%\right)$ for 2501 observed reflections with $I \geq 3 \sigma(I)$. The function minimized during refinement was $\sum w\left(\left|F_{o}\right|-\left|F_{c}\right|\right)^{2}$, where $w=1 /$ $\left[\sigma^{2}(F)+0.001\left(F^{2}\right)\right]$.

Fractional coordinates and related data are available as supplementary material.* Bond lengths and bond angles do not show significant or systematic differences from expected values. Conformational angles and hydrogen-bond parameters are listed in Table II and Table III, respectively.

* Supplementary materials consisting of fractional coordinates for the nonhydrogen atoms, bond lengths, bond angles, anisotropic thermal parameters, and coordinates for $\mathrm{H}$ atoms will be deposited with the Cambridge Structural Data Base, University Chemical Laboratory, Lensfield Road, Cambridge CB21EW, UK. Coordinates are also available from the authors on request. 
Table II Torsion angles ${ }^{\text {a }}$ (deg) of Boc-Gly-GlyLeu-Aib-Val-OMe

\begin{tabular}{lrrrrr}
\hline Residue & $\phi$ & $\psi$ & $\omega$ & $\chi^{1}$ & $\chi^{2}$ \\
\hline Gly & $-92^{\mathrm{b}}$ & -133 & 172 & & \\
Gly & 140 & 170 & 176 & & \\
Leu & -61 & 130 & 179 & -70 & $-76,161$ \\
Aib & 71 & 6 & -176 & & \\
Val & -91 & $133^{\mathrm{c}}$ & $176^{\mathrm{d}}$ & $-65,171$ & \\
\hline
\end{tabular}

${ }^{\mathrm{a}}$ The torsion angles for rotation about bonds of the peptide backbone $(\phi, \psi$, and $\omega)$ and about bonds of the amino acid side chains $\left(\chi^{1}, \chi^{2}\right)$ as suggested by the IUPAC-IUB Commission on Biochemical Nomenclature (1970). Estimated standard deviations $\sim 1.0^{\circ}$.

${ }^{\mathrm{b}} \mathrm{C}^{\prime}(0)-\mathrm{N}(1)-\mathrm{C}^{\alpha}(1)-\mathrm{C}^{\prime}(1)$

${ }^{\mathrm{c}} \mathrm{N}(5)-\mathrm{C}^{\alpha}(5)-\mathrm{C}^{\prime}(5)-\mathrm{O}(5)$.

${ }^{\mathrm{d}} \mathrm{C}^{\alpha}(5)-\mathrm{C}^{\prime}(5)-\mathrm{O}(6)-\mathrm{C}(6)$.

\section{RESULTS AND DISCUSSION}

\section{Crystal Structure}

A stereo view of the molecular conformation of the pentapeptide Boc-Gly-Gly-Leu-Aib-Val-OMe is shown in Figure 1. Inspection of the backbone torsion angles in Table II establishes that the Leu(3)Aib (4) segment adopts a type II $\beta$-turn conformation with a $4 \rightarrow 1$ hydrogen bond between the Gly (2) $\mathrm{CO}$ and $\mathrm{Val}(5) \mathrm{NH}$ groups (Table III). The $\phi, \psi$ values of the Gly (1) and Gly(2) residues lie in the extended region of conformational space. $\operatorname{Gly}(1)\left(\phi=-92^{\circ}, \psi=-133^{\circ}\right)$ adopts a conformation generally observed in extended $\beta$-strands that form part of $\beta$-sheets, but Gly (2) ( $\phi=140^{\circ}, \psi$ $=170^{\circ}$ ) adopts a more fully extended conformation. The $\operatorname{Val}(5)\left(\phi=-91^{\circ}, \psi=133^{\circ}\right)$ residue also adopts an extended conformation. The molecular packing in the crystal is shown in Figure 2. All the five $\mathrm{NH}$ groups in the molecule participate in hydrogen bonding with $\mathrm{CO}$ groups, with four intermolecular hydrogen bonds being formed between symmetry-related molecules (Table III). The only potential hydrogen-bonding function that is not satisfied is the $\mathrm{CO}$ moiety of the Bocprotecting group.

\section{Comparison of the Conformation Observed for the Pentapeptide Segment}

Dramatically different conformations for the GlyGly-Leu-Aib-Val segment have been observed in the crystal structure of a protected pentapeptide (this study) and a protected octapeptide (BocLeu-Aib-Val-Gly-Gly-Leu-Aib-Val-OMe ${ }^{3}$ ). Figure 3 compares the molecular conformations observed in the two structures. In the octapeptide the $\operatorname{Gly}(4)\left(\phi=-65^{\circ}, \psi=-23^{\circ}\right)$, Gly(5) $\left(\phi=-64^{\circ}\right.$, $\left.\psi=-24^{\circ}\right)$, Leu $(6)\left(\phi=-81^{\circ}, \psi=-16^{\circ}\right)$, and $\operatorname{Val}(8)\left(\phi=-59^{\circ}, \psi=-39^{\circ}\right)$ residues adopt right-handed helical conformations while the Aib(7) residue adopts a left-handed helical conformation $\left(\phi=+53^{\circ}, \psi=+49^{\circ}\right)$. The observed conformation resembles a $3_{10}$-helix, with a helix terminating $6 \rightarrow 1$ hydrogen bond that is a consequence of the helix reversal at Aib(7). In sharp contrast, in the pentapeptide the Leu(3)-Aib(4) segment forms a type-II $\beta$-turn, with the Gly (1), Gly(2), and Val(5) residues being extended. In-

Table III Hydrogen Bonds of Boc-Gly-Gly-Leu-Aib-Val-OMe

\begin{tabular}{|c|c|c|c|c|c|c|c|}
\hline \multirow[b]{2}{*}{ Type } & \multirow[b]{2}{*}{ Donor } & \multirow[b]{2}{*}{ Acceptor } & $\mathrm{N}-\mathrm{O}$ & $\mathrm{H}-\mathrm{O}$ & $\mathrm{C}=\mathrm{O}-\mathrm{N}$ & $\mathrm{O}-\mathrm{HN}$ & \multirow[b]{2}{*}{ Symmetry } \\
\hline & & & \multicolumn{2}{|c|}{ Length ( } & \multicolumn{2}{|c|}{ Angle (deg) } & \\
\hline \multicolumn{8}{|c|}{ Intermolecular } \\
\hline & $\mathrm{N}(1)$ & $\mathrm{O}(4)^{\mathrm{a}}$ & 2.97 & 2.21 & 153 & 125 & $1+x, y, z$ \\
\hline & $\mathrm{N}(2)$ & $\mathrm{O}(3)^{\mathrm{a}}$ & 2.98 & 1.94 & 157 & 158 & $-1-x,+1 / 2+y,-z$ \\
\hline & $\mathrm{N}(3)$ & $\mathrm{O}(5)^{\mathrm{a}}$ & 3.07 & 2.01 & 156 & 165 & $-1-x,-1 / 2+y,-z$ \\
\hline & $\mathrm{N}(4)$ & $\mathrm{O}(1)^{\mathrm{a}}$ & 2.87 & 1.9 & 156 & 159 & $-1-x,+1 / 2+y,-z$ \\
\hline \multicolumn{8}{|c|}{ Intramolecular } \\
\hline & $\mathrm{N}(5)$ & $\mathrm{O}(2)$ & 3.03 & 2.02 & 127 & 155 & \\
\hline
\end{tabular}

${ }^{\text {a }}$ Symmetry related as shown in the last column to the molecule whose coordinates have been deposited. 


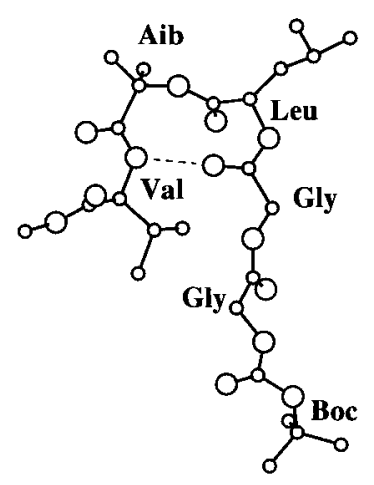

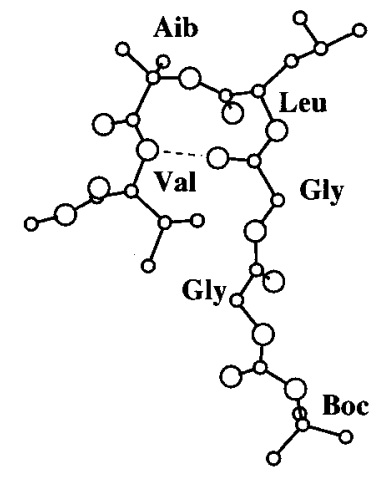

FIGURE 1 Stereo view of the pentapeptide in the crystal. The single intramolecular hydrogen bond has been indicated by a broken line.

terestingly, nmr studies of the octapeptide in dimethylsulfoxide strongly suggest unfolding of the helix with type-II $\beta$-turn formation at the Leu (6)-
Aib (7) segment. ${ }^{3}$ This suggested solution conformation coincides with the crystalline conformation observed in the pentapeptide. It is thus clear that the intrinsic conformational flexibility of the Gly-Gly segment is manifested in the remarkably different folding pattern observed in the two peptides. It is also noteworthy that the Leu-Aib segment adopts a type II $\beta$-turn conformation in preference to a type III $\beta$-turn conformation $\left(\phi_{i+1}\right.$ $=-60^{\circ}, \psi_{i+1}=-30^{\circ}$, and $\phi_{i+2}=-60^{\circ}, \psi_{i+2}$ $\left.=-30^{\circ}\right)$. The heterogeneity of the conformations of Gly-Gly segments in peptide crystal structures is also illustrated by the variety of conformations characterized in enkephalins and oligopeptides containing contiguous glycine residues. Table IV provides a summary. The most common conformations observed appear to be $\beta$-turn and fully extended structures, a feature also noted in the present structural comparison between pentapeptide and octapeptide structures. A search for GlyGly segments in a database of 250 protein crystal

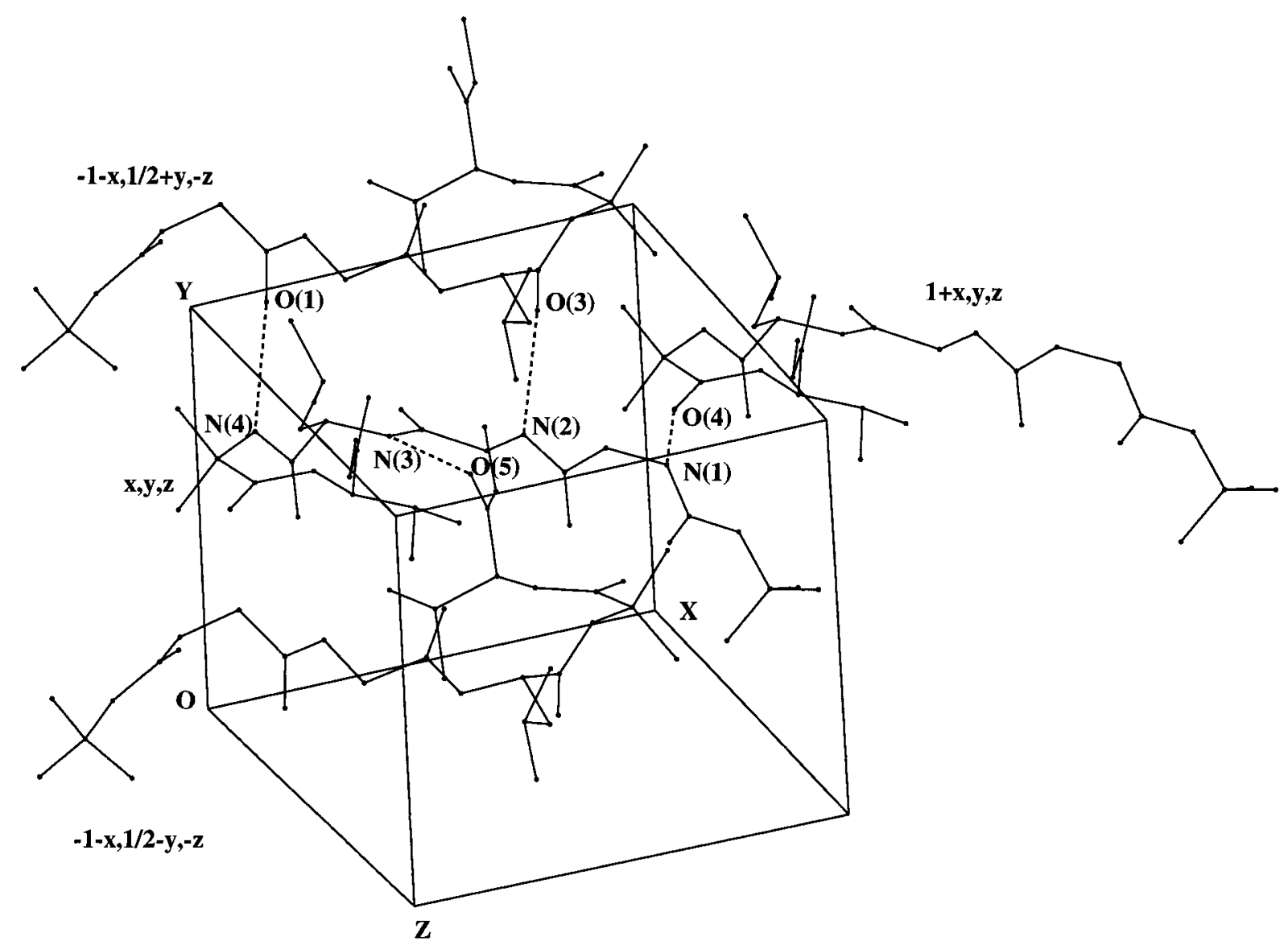

FIGURE 2 Molecular packing in the crystal showing only the intermolecular hydrogen bonds. 


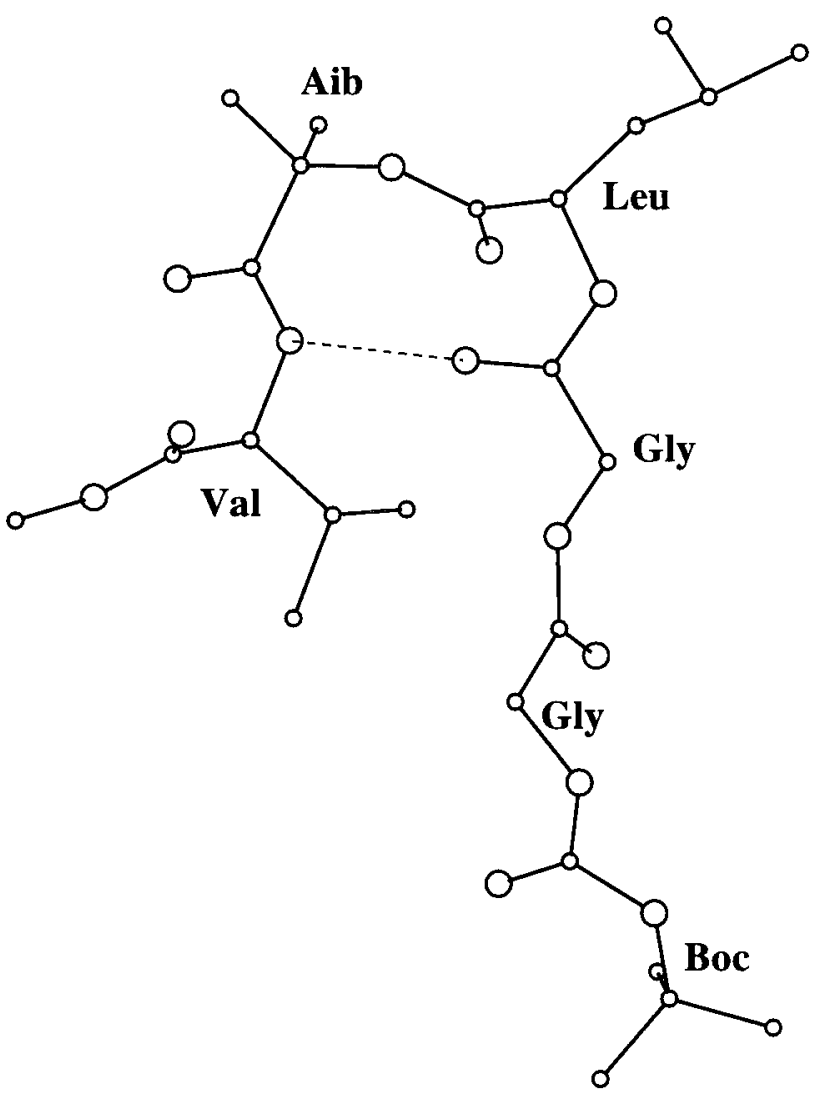

A

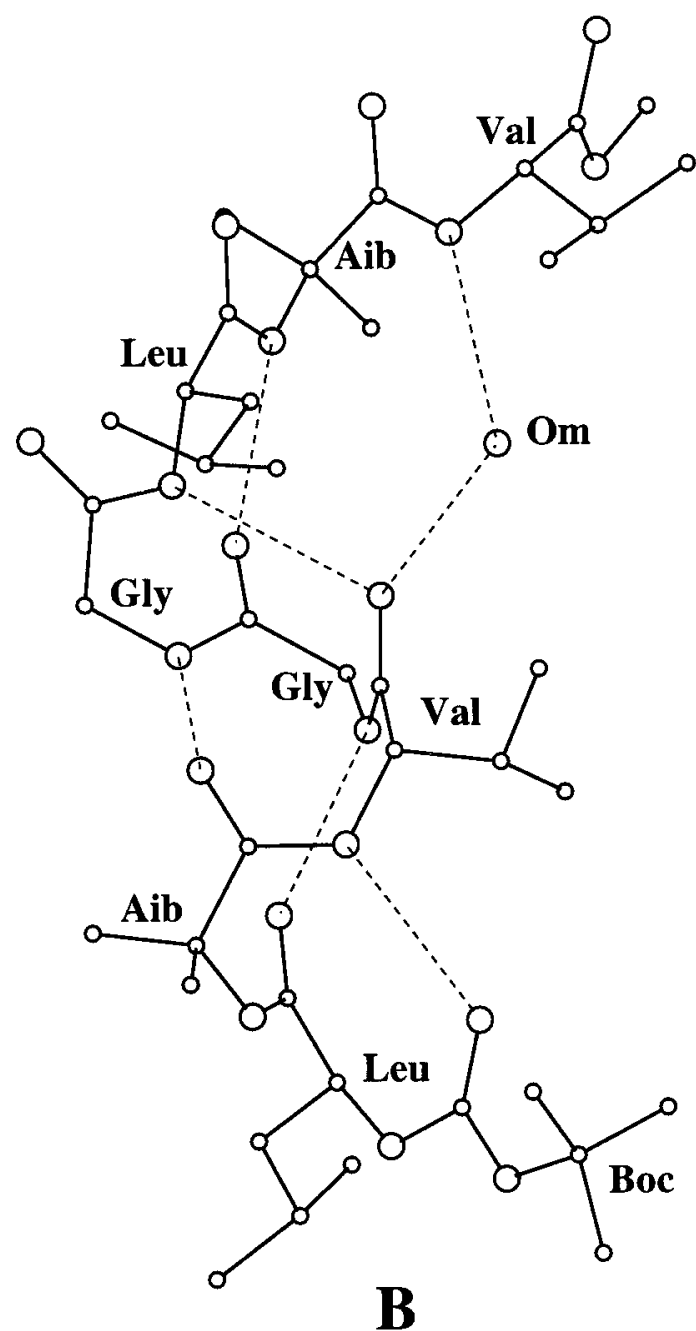

FIGURE 3 A comparative view of the molecular conformations of the pentapeptide (A) and the octapeptide (B). Om indicates $\mathrm{CH}_{3} \mathrm{OH}$.

structures, refined at resolutions $\leq 2.0 \AA$ and with homology less than $40 \%$, revealed 363 examples. Of these, 100 examples form part of secondary structures with the following distributions: 29 in helices, 13 in $\beta$-strands, and 58 in $\beta$-turns. The remaining 263 examples are part of irregular elements with a large fraction consisting conformations that are largely extended (K. Gunasekaran and S. Datta, unpublished).

The comparison of the crystal structures of the pentapeptide Boc-Gly-Gly-Leu-Aib-Val-OMe and the octapeptide Boc-Leu-Aib-Val-Gly-GlyLeu-Aib-Val-OMe reveal dramatically different conformations for the Gly-Gly segment. It is clear that in peptides that contain potentially flexible segments, the introduction of conformationally constrained residues may result in stabilization of specific folded conformations. However, such sequences may be poised to undergo conformational transitions, which may be introduced either by variation in solvation or by changes in peptide length or sequence. Crystal structure determinations, as in the present study, can firmly characterize the detailed stereochemistry of the possible conformational states and may provide a starting point for computer simulation of conformational transitions.

We are grateful to K. Gunasekaran for sharing the results of an analysis of Gly-Gly segments in proteins. This re- 
Table IV Backbone Conformational Angles for Gly-Gly Segments in Representative Peptide Crystals

\begin{tabular}{|c|c|c|c|c|c|}
\hline \multirow[b]{2}{*}{ Peptides } & \multicolumn{4}{|c|}{$\begin{array}{c}\phi \text { and } \psi \text { of Gly-Gly Segment } \\
\text { (deg) }\end{array}$} & \multirow[b]{2}{*}{ References } \\
\hline & $\phi(1)$ & $\psi(1)$ & $\phi(2)$ & $\psi(2)$ & \\
\hline Boc-Gly-Gly-Leu-Aib-Val-OMe & -92 & -133 & 140 & 170 & This study \\
\hline Boc-Leu-Aib-Val-Gly-Gly-Leu-Aib-Val-OMe & -65 & -23 & -64 & -24 & 3 \\
\hline Tyr-Gly-Gly-Phe & 74 & 11 & 90 & -40 & 8 \\
\hline H-Tyr-Gly-Gly-Phe-Leu-OH & 59 & 25 & 97 & -7 & 9 \\
\hline Boc-Gly-Gly-Phe-OC ${ }_{2} \mathrm{H}_{5}$ & 98 & 9 & 109 & 62 & 10 \\
\hline H-Tyr-Gly-Gly-Phe-Leu-OH ${ }^{\mathrm{a}}$ & & & & & 11 \\
\hline Molecule A & -144 & 114 & -122 & 132 & \\
\hline Molecule B & 151 & -155 & 154 & -151 & \\
\hline Molecule C & 141 & -157 & 174 & -170 & \\
\hline Molecule D & -131 & 142 & -144 & 131 & \\
\hline Boc-Tyr-Gly-Gly-(4-bromo)Phe-Met-OH & -175 & -175 & 143 & -157 & 12 \\
\hline H-Tyr-Gly-Gly-(4-bromo)Phe-Leu-OH & 59 & 27 & 94 & -6 & 13 \\
\hline H-Leu-Gly-Gly-Gly-OH & 106 & -2 & -59 & 140 & 14 \\
\hline H-Tyr-Gly-Gly-(4-bromo)Phe-Met-OH & & & & & 15 \\
\hline Molecule A & -170 & 166 & -159 & 164 & \\
\hline Molecule B & 172 & -166 & -164 & -169 & \\
\hline H-Tyr-Gly-Gly-Phe-Met-OH & & & & & 15 \\
\hline Molecule A & -165 & 163 & -163 & 160 & \\
\hline Molecule B & 177 & -174 & 170 & -173 & \\
\hline H-Tyr-Gly-Gly-Phe-Leu-OH & -59 & -34 & -63 & -24 & 16 \\
\hline
\end{tabular}

${ }^{\text {a }}$ Four molecules in the asymmetric unit indicated as molecules A, B, C, and D, respectively. Two molecules in the asymmetric unit labeled as molecules A and B.

search was supported by the Department of Science and Technology, Government of India. SD acknowledges receipt of a Senior Research Fellowship from the University Grants Commission.

\section{REFERENCES}

1. Karle, I. L. (1989) Biopolymers 28, 1-14.

2. Gurunath, R. \& Balaram, P. (1995) Biopolymers 35, 21-29.

3. Karle, I. L., Banerjee, A., Bhattacharjya, S. \& Balaram, P. (1996) Biopolymers, 38, 515-526.

4. Prasad, B. V. V. \& Balaram, P. (1984) CRC Crit. Rev. Biochem. 16, 307-348.

5. Karle, I. L. \& Balaram, P. (1990) Biochemistry 29, 6748-6756.

6. Debaerdemaeker, T., Germain, G., Main, P., Tate, C. \& Woolfson, M. M. (1987) MULTAN-87, A System of Computer Programs for the Automatic Solution of Crystal Structures from X-ray Diffraction Data, University of York, England.

7. Sheldrick, G. M. (1976) SHELX-76, Program for
Crystal Structure Determination, Cambridge University, UK.

8. Fournie Zaluski, M.-C., Prange, T., Pascard, C. \& Roques, B. P. (1977) Biochem. Biophys. Res. Commun. 79, 1199-1206.

9. Smith, G. D. \& Griffin, J. F. (1978) Science 199, 1214-1216.

10. Ishida, T., Tanabe, N. \& Inoue, M. ( 1983 ) Acta Crystallogr. Sect. C 39, 110-112.

11. Camerman, A., Mastropaolo, D., Karle, I. L., Karle, J. \& Camerman, N. (1983) Nature (London) 306, 447-450.

12. Doi, M., Ishida, T., Inoue, M., Fujiwara, T., Tomita, K., Kimura, T. \& Sakakibara, S. (1984) FEBS Lett. 170, 229-231.

13. Ishida, T., Kenmotsu, M., Mino, Y., Inoue, M., Fujiwara, T., Tomita, K., Kimura, T. \& Sakakibara, S. (1984) Biochem. J. 218, 677-689.

14. Srikrishnan, T. \& Parthasarathy, R. (1987) Int. J. Peptide Protein Res. 30, 557-563.

15. Doi, M., Tanka, M., Ishida, T., Inoue, M., Fujiwara, T., Tomita, K., Kimura, T., Sakakibara, S. \& Sheldrick, G. M. (1987) J. Biochem. 101, 485-490.

16. Aubry, A., Birlirakis, N., Sakarellos-Daitsiotis, M., Sakarellos, C. \& Marraud, M. (1989) Biopolymers 28, 27-40. 